University at Albany, State University of New York

Scholars Archive

\title{
Psychotherapy Clients' Recalled Treatment Experiences: A Survey of Perceived Evidence-Based Practice Elements
}

Yadi Chen

University at Albany, State University of New York, ychen@albany.edu

Follow this and additional works at: https://scholarsarchive.library.albany.edu/honorscollege_psych

Part of the Psychology Commons

\section{Recommended Citation}

Chen, Yadi, "Psychotherapy Clients' Recalled Treatment Experiences: A Survey of Perceived EvidenceBased Practice Elements" (2017). Psychology. 11.

https://scholarsarchive.library.albany.edu/honorscollege_psych/11

This Honors Thesis is brought to you for free and open access by the Honors College at Scholars Archive. It has been accepted for inclusion in Psychology by an authorized administrator of Scholars Archive. For more information, please contact scholarsarchive@albany.edu. 


\title{
Psychotherapy Clients' Recalled Treatment Experiences: A Survey of Perceived Evidence-Based Practice Elements
}

\author{
An Honors Thesis presented to the \\ Department of Psychology, \\ University at Albany, State University of New York \\ in partial fulfillment of the requirements \\ for graduation with Honors in Psychology
}

\section{Yadi Chen}

Research Advisor: Dr. James Boswell

Second Reader: Dr. Robert Rosellini 


\begin{abstract}
Background: Common evidence-based practice (EBPs) elements can be observed across cognitive-behavioral therapy (CBT) manuals for commonly occurring disorders. Example EBP elements include exposure, cognitive restructuring, teaching active coping skills (e.g., relaxation), enhancing positive affect, and facilitating a positive working alliance. It is unclear if EBP elements are frequently delivered or prioritized in routine psychotherapy. Also, little is known about the prevalence or pervasiveness of EBP elements from the routine clients' perspective. Objective: The objective of this study was to assess psychotherapy clients' self-reported retrospective treatment experiences with regard to common EBP elements. Method: Participants $(\mathrm{N}=592)$ were consenting university undergraduate students who received course credit. Eligibility: (a) 18 years of age or older, and (b) have current or previous experience with psychotherapy or counseling. The sample was mostly female (58.1\%) and Caucasian (54.4\%), with a mean age of 19.04 years $(S D=2.44)$. Eligible participants were invited to access a web-based survey that included (a) diverse measures of current symptoms and functioning; (b) an item assessing the problem domain(s) of focus during their therapy; and (c) the presence/absence of 8 potential EBP elements and the extent to which they were discussed during/were a focus of their psychotherapy. Results: Overall, routine psychotherapy clients recalled receiving a variety of EBP elements, the most common being a focus on positive emotions. The presence or absence of a recalled EBP element was, in some cases, associated with endorsement of a specific problem domain. The recall of receiving an EBP element was not consistently related to better current functioning. Implications: Future research should continue to focus on clients' experience of psychotherapy elements, including what was/is most and least helpful to assist in the refinement and implementation of EBPs.
\end{abstract}




\section{Acknowledgments}

First, I would like to express my sincere gratitude to my research advisor Dr. James Boswell for the continuous support of my honors project, for his intelligence, motivation, and immense knowledge. Thank you for the advice and willingness that allowed me to pursue research on topics for which I am truly passionate. Your guidance helped me in all the time of research and writing of this thesis.

I would like to thank Dr. Robert Rosellini for being supportive over my undergraduate career. Thank you for believing in me. Also, I want to thank Dr. Leslie Halpern for giving me a lot of inspiration on how to become a better student. You certainly are a great mentor for me and you have been very generous in sharing your valuable knowledge in the clinical psychology field. I absolutely must thank Dean Hui-Ching Chang, for all the support you gave me and all the wonderful academic discussions we had.

Last but not least, I would like to thank my parents, whose guidance and love are with me in whatever I pursue. 
Psychotherapy Clients' Recalled Treatment Experiences: A Survey of Perceived Evidence-Based

\section{Practice Elements}

Research has demonstrated the efficacy of psychological treatments for common psychiatric disorders (Nathan \& Gorman, 2015), and that common "affective" disorders share etiological and maintaining mechanisms (Barlow et al., 2014). It is widely recognized in the field of psychotherapy that psychotherapy delivered in routine, everyday treatment settings can differ from the psychotherapy being delivered in research protocols and the strategies codified in manuals (Goisman, Warshaw, \& Keller, 1999). Undoubtedly, there is frequently a gap between psychotherapy research-vetted practices and routine clinical practice (Kazdin, 2008). For example, cognitive behavioral therapies (CBT) include intervention components that have been empirically supported for the treatment of eating disorders (Friedman et al., 2016), yet a survey of clinicians demonstrated that CBT strategies were not routinely used with eating disorder clients (Waller, Stringer, \& Meyer, 2012). The implementation of research-informed practice plays an important role in the quality of client care and public health broadly (Brownson, Colditz, \& Proctor, 2012).

Although the inconsistent use of evidence-based practices (EBPs) is widely recognized, it can be difficult to assess the nature of psychotherapy being delivered in routine service settings. Such interventions are delivered across diverse settings by diverse practitioners from diverse training programs (Lambert, 2013), and confidentiality is critical. Gold standard assessment strategies involve independent observations of within-session activities, and this is not feasible in 
most settings (Garland, \& Schoenwald, 2013). With some exceptions (e.g., Stirman, Gutner, Crits-Christoph, \& Beidas, 2015), existing studies have relied on psychotherapist self-report (e.g., Waller et al., 2012). Focused specifically on CBT for eating disorders, Waller et al. (2012) found that many clinicians who identified themselves as CBT practitioners did not endorse significant, consistent use of specific CBT interventions.

Most studies in this area, including Waller et al. (2012), focus on diagnosis-specific techniques. However, there are many examples of evidence-based treatment strategies that cut across diagnoses and models (Boswell, 2013), and such research support spans both basic psychopathology and applied clinical research (Castonguay \& Oltmanns, 2013). According to the American Psychological Association (2005), EBP is the integration of the best available research with clinical expertise. The complexity of this issue is underscored by a recent government sponsored task force report on developing a framework for establishing EBP standards (England, Butler, \& Gonzalez, 2015). This task force largely advocated for an "elements" approach to EBP standards, based on the argument that EBP cannot be reduced to the frequency of specific technique use. Their developed framework divided EBP elements into (a) unique specific, (b) shared specific, and (c) common elements. For example, prolonged imaginal exposure is a unique (CBT) specific intervention element for PTSD (Foa, Chrestman, \& Gilboa-Schechtman, 2008); other treatment approaches involve strategies to explore and reduce attempts to avoid distressing thoughts and feelings (shared element); and effective practice involves strategies to engage the client in the treatment (common element) (England et al., 2015). 
Many similar examples can be identified within and between disorders and problem domains. In addition, by following the above framework, research findings may point to psychotherapy problems and strategies that are not adequately addressed in existing manualized treatments. For example, multiple areas of research point to low levels of positive affect in social anxiety disorder (Brown \& Barlow, 2009). Relatively stronger associations with low levels of positive affect differentiate sociality anxiety disorder from other anxiety disorders in its similarity with major depression (Brown, 2007). Yet few existing manualized therapies for social anxiety explicitly identify positive emotion as a target of intervention. Another example can be found in the common factors literature. Extant research has demonstrated a robust association between the quality of the client-therapist working alliance and treatment outcome (Horvath, Del Re, Flückiger, \& Symonds, 2011). Contemporary treatment manuals reference the importance of the working alliance for engagement and outcome (e.g., Barlow et al., 2011), yet few commonly used manuals include specific recommendations for alliance-enhancement and repair (in the case of alliance problems) strategies (Castonguay, Constantino, Boswell, \& Kraus, 2010). As a common EBP element, little is known about the extent to which "frontline" clinicians attend to the working alliance in their routine treatment provision.

It is also important to once again highlight that existing work on the implementation of EBP elements in routine practice has relied on independent observation of relatively small samples, due to logistical constraints, or therapist self-report. The field has become increasingly interested in client-centered care and prizing the client's perspective as it relates to treatment 
process and decision making (Castonguay et al., 2010; Fisher \& Boswell, 2016). We are unaware of research that has directly surveyed psychotherapy and counseling client's perceptions of the presence/absence, and the degree to which, EBP elements were a focus of their treatment. In order to examine what is and is not happening in routine psychotherapy from the client's perspective, this study examined current and former psychotherapy participants' recollections of what took place during their therapy as it pertains to select EBP elements. If participants endorse the presence of EBP elements in their psychotherapies, this may imply that community psychotherapists are actually practicing in a manner that is consistent with EBP elements, yet in a manner that may not fit neatly into traditional conceptualizations of EBP as reducible to unique specific protocol techniques. If participants never or rarely endorse the presence of the EBP elements, this might imply that the gap between research and practice does not exist purely at the level of unique specific elements; rather, even shared and common EBP elements may be underutilized.

\section{The Present Study}

We conducted a web-based survey of self-identified psychotherapy and counseling clients' recalled treatment experiences. In order to be more generalizable, we elected to focus on shared specific and common EBP elements in this study. A large number of such elements can potentially be identified. In part due to feasibility concerns, we selected eight elements that might be reasonably implemented across diverse routine psychotherapies for the most common problems (e.g., anxiety and depression, relationship concerns), and that are consistent with the 
intervention principles identified by Castongay and Beutler (2006). In addition, it was important for the element descriptors to be jargon-neutral to ease interpretation and avoid potential confusion. The selected elements included: (1) attending to positive emotions, (2) facilitating motivation to change, (3) addressing the working alliance, (4) encouraging confrontation of difficult or avoided situations, thoughts, and feelings, (5) focusing on thinking patterns, (6) implementing relaxation training, (7) addressing social skills, and (8) focusing on stress management.

In addition to the presence or absence of a specific EBP element, if endorsed, participants were asked to rate the degree to which these issues and strategies were a focus of their routine psychotherapy and counseling. In order to provide additional context for interpreting the levels of endorsement (or lack thereof), the study also included questions regarding the nature of the participants' problems that led them to seek psychotherapy, as well as participant characteristics (e.g., age, ethnicity), and current symptoms and functioning.

The primary aim of this study was largely descriptive. We were first and foremost interested in the frequency and degree of endorsement of the EBP elements. We were then interested in the factors that might be associated with the presence/absence and the degree to which an element was a focus of routine treatment from the client's perspective. The latter interest focused on the following questions: (a) is identified gender associated with EBP element endorsement and/or the degree of recalled focus?; (b) is identified ethnicity associated with EBP element endorsement and/or the degree of recalled focus?; (c) is identified problem domain 
associated with EBP element endorsement and/or the degree of recalled focus?; and (d) is the endorsement of an EBP element associated with current symptoms and functioning? Given the absence of empirical findings in this area, this work was largely exploratory and we did not formulate directional hypotheses.

\section{Method}

\section{Participants and Procedures}

All study procedures and materials were approved by the University at Albany, SUNY institutional review board (IRB). Participants were recruited online through the psychology department Research Pool and through posted flyers. All consent and questionnaire completion was web-based and conducted outside of the lab. All participants provided informed consent prior to participation. Participants were University at Albany undergraduate students who were enrolled in a psychology course, and they received course credit for participation. Eligible, participants needed to be (a) 18 years of age or older, and (b) endorse experience receiving psychotherapy or counseling (past and/or current). Individuals who had no experience with therapy or counseling were excluded.

A total of $\mathrm{N}=592$ eligible individuals began the assessment and completed a sufficient number of assessment items to be included in primary analyses. Missing data were present for many cases, most instances at the item level. A missing value analysis indicated that percent missing individual item responses were within acceptable limits to be considered missing at random and appropriate for item-level value estimation (Tabachnick \& Fidell, 2013). Item-level 
missing values were estimated using the expectation-maximization (EM) algorithm in SPSS 23 (Dempster, Laird, \& Rubin, 1977). No significant item mean differences were observed between the cases with only observed and cases with observed and estimated values. Analyses were performed with the data set with estimated values. Missing values were not estimated for demographic items, EBP element items, or when all scale items were missing for a case. Listwise deletion was used as the default in such cases, so the sample size varied among analyses.

The mean participant age was 19.04 years $(\mathrm{SD}=2.44$, range $=18$ to 57 years). The sample was mostly female $(58.1 \%, \mathrm{n}=344)$ and Caucasian $(54.4 \%, \mathrm{n}=322)$. The most common academic standing was freshmen $(62 \%, n=367)$, followed by sophomores $(21.5 \%, n=127)$, juniors $(9.6 \%, \mathrm{n}=57)$, and seniors $(6.9 \%, \mathrm{n}=41)$.

\section{Measures}

Participants completed the study questions via the secure online platform Qualtrics. In addition to informed consent, the assessment battery included the following measures.

\section{Demographic Questionnaire}

Participants first completed a set of demographic items that asked about age, sex, ethnicity, and academic standing. In addition, participants were asked to identify the problems that were the primary focus of their psychotherapy. Problem comorbidity is common (Brown, Campbell, Lehman, Grisham, \& Mancill, 2001), so participants could select more than one domain.

\section{Current Symptoms and Functioning}


Liebowitz Social Anxiety Scale (LSAS; Liebowitz, 1987). The LSAS was used to assess symptoms related to social anxiety disorder. This scale includes 24 items, and these items are divided into two subscales: 13 questions concern performance anxiety and 11 relate to avoidance of social situations. The total score was used in the present study. The LSAS has demonstrated reliability and validity in previous research (Heimberg et al., 1999).

Dimensional Obsessive-Compulsive Scale (DOCS; Abramowitz, 2010). The DOCS is a 20 -item self-report instrument that measures the severity of obsessive compulsive disorder symptoms. Participants were asked about experiences with thoughts and behaviors related to (1) contamination, (2) harm and disasters, (3) symmetry, (4) unwanted thoughts. The total score was used in the present study. The DOCS has demonstrated reliability and validity in previous research (Abramowitz et al., 2010)

Generalized Anxiety Disorder-7 Scale (GAD-7; Spitzer et al., 2006). The 7-item self-report measure is commonly used to assess general anxiety symptoms, including worry. Item ratings are summed for a single overall score. Example items include: "Feeling nervous, anxious, or on edge" and "Not being able to stop or control worrying." The GAD-7 has demonstrated reliability and acceptable convergent validity (Spitzer et al., 2006).

Panic Disorder Severity Scale-Self Report (PDSS-SR; Shear et al., 2001). The PDSS was used to evaluate panic disorder symptoms. It consists of 5 items, and each item is rated on a 5-point scale that ranges from 0 to 4 . The total score was used in this study. The PDSS-SR has demonstrated adequate reliability and validity in previous research (Boswell et al., 2013; Shear et 
al., 2001).

Patient Health Questionnaire (PHQ-9; Kroenke et al., 2001). The widely used PHQ-9 was used to assess depression symptoms Example items include "Little interest or pleasure in doing things," and "Feeling down, depressed or hopeless." The total score was used in this study. The PHQ-9 has demonstrated good reliability and validity in previous research (Kroenke et al., 2001).

Positive and Negative Affect Schedule (PANAS; Watson et al., 1988). This 20-item self-report measure assesses the frequency of positive and negative affect (e.g., interested, distressed, excited, upset). Both the positive affect and negative affect subscale items are scored on a scale of 1 (very slightly or not at all) to 5 (Extremely), with higher scores indicating higher levels of each. The PANAS has demonstrated good psychometric properties in previous research (Watson et al., 1988).

Inventory of Interpersonal Problems (IIP-32; Horowitz et al., 2000). This 32-item self-report instrument assesses the severity and nature of interpersonal problems. A total score can be derived as an index of overall interpersonal distress, and this was used in the present study. Items are worded as a potential problem of engaging in something too much (doing it too frequently and hard to stop) or too little (should be doing it more frequently but hard to do). Example items include "It is hard for me to understand another person's point of view," and "I am too aggressive toward other people." Participants were asked to rate on a 5-point scale that ranges from 0 to 4 . The 32-item IIP has demonstrated reliability and validity in previous research 
(Horowitz et al., 2000).

Subjective Vitality Scale (SVS; Ryan \& Frederick, 1997). The SVS consists of 7 items, each with a range from 1 (not at all true) to 7 (very true). SVS was used to evaluate one's level of vitality as an indicator of positive mental health. Example items include "I feel alive and vital" and "I don't feel very energetic." The total score was used in this study and previous research has demonstrated good reliability and validity for this measure (Ryan \& Frederick, 1997).

Flourishing Scale (FS; Diener \& Biswas-Diener, 2009). This 8-item self-report measure was used to evaluate psychological resources and strengths. Within each item, participants rate the self-perceived success in areas (e.g., "I lead a purposeful and meaningful life" and "My social relationships are supportive and rewarding"). Participants were asked to indicate their agreement with each item by using the 1-7 scale (1 [strongly disagree] to 7 [strongly agree]). A higher score indicates individual with better psychological health and well-being. This measure has demonstrated good psychometric properties in previous research (Diener \& Biswas-Diener, 2009).

\section{Evidence-Based Practice Element Items}

For the purposes of this study, we created eight, two-part items that assessed (1) the presence or absence of an element, and (2) the degree of perceived focus on that element during treatment. For example: "Did your therapist or counselor address or talk about the importance of positive emotions during your therapy or counseling?" If the participant responded 'yes' to the entry question, they were then asked to rate the extent of focus from 1 (rarely) to 4 (extensively 
[the main focus of what we did]). If a participant responded 'no,' then they went on to the next element item.

\section{Results}

\section{Descriptives}

The most frequently endorsed problem domain (domain for which they sought help and was a focus of the psychotherapy) was relationship or family problems (51\%), followed by generalized anxiety/excessive worry $(40.7 \%)$, depression $(40.0 \%)$, social anxiety $(21.1 \%)$, panic (10.1\%), eating (7.9\%), obsessions and compulsions $(6.4 \%)$, and substance use $(4.4 \%)$. The mean total score, standard deviation, possible range, and internal consistency estimate for the sample is reported for each symptom and functioning self-report scale in Table 1.

EBP element frequencies and degree of focus (if endorsed) means and standard deviations are reported in Table 2 . The most frequently endorsed elements were attention to positive emotions and confrontation of feared and/or avoided situations (i.e., exposure). The least frequently endorsed elements (below 50\%) were a focus on the relationship/working alliance and the implementation of social skills training. Overall, little variability was observed across elements with regard to the extent of the focus on an element. The average scores were between 2.03 (relationship) and 2.54 (stress management), which is between occurring "on multiple occasions" (2) and "frequently" (3).

\section{Associations with Demographic Factors and Problem Domains}

The associations between EBP element endorsement (presence/absence coded 
dichotomously) and demographic characteristics and identified problem domains, respectively, were examined with chi square tests. In addition, the categorical demographic and problem domain variables were entered as between-subjects factors in separate one-way ANOVAs predicting degree of EBP element focus (for participants who endorsed the presence of the element). In order to decrease the number of follow-up tests, the ethnicity scores were recoded to be dichotomous (racial/ethnic majority vs. non-majority).

The results for the demographic factors of sex and ethnicity are reported in Table 3. Results indicated that identified females were more likely than males to recall a focus on confronting avoidance and stress management as occurring in their psychotherapy. Identified males were more likely than females to endorse receiving social skills training. Identifying minority participants were more likely to report focusing on motivation enhancement and social skills training compared to identifying ethnic majority individuals. Interestingly, differences in the presence of a particular EBP element were not necessarily consistent with differences in the degree of emphasis if the element was recalled as being present. For example, male and female clients did not differ on the presence or absence of focusing on the working relationship, yet when this element was recalled as being present, male clients endorsed a stronger degree of emphasis than females. When present, female clients reported a stronger degree of emphasis on confronting avoidance than male clients. When present, ethnic minority clients recalled psychotherapies with a greater focus on motivation and the working relationship than ethnic majority clients. Some problem domains were also associated with presence of EBP elements, as 
well as the degree of focus.

\section{Problem Domains and EBP Elements}

Depression. Individuals who endorsed depression as a key problem focus of their psychotherapy were not significantly more likely to report attention to positive emotions $\left(\chi^{2}(1)\right.$ $=.07, p=0.79)$, yet for those who did report that this was present $(82.7 \%)$, endorsing depression was associated with stronger recalled emphasis ratings, $F(1,466)=7.05, p<.01$. Endorsement of depression was also associated with greater likelihood of recalling motivation enhancement $\left(79.0 \%, \chi^{2}(1)=4.52, p<.05\right)$; conversely, this was associated with lower likelihood of recalling a focus on the working alliance $\left(44.7 \%, \chi^{2}(1)=4.36, p<.05\right)$. Endorsement of depression was significantly associated with both the endorsement $\left.\left(75.0 \%, \chi^{2}(1)=4.01\right), p<.05\right)$ and the degree of focus $(F(1,391)=11.4, p<.01)$ on thinking patterns, as well as the endorsement $\left(78.4 \%, \chi^{2}(1)\right.$ $=7.75), p<.05)$ and the degree of focus $(F(1,397)=10.70, p<.01)$ on stress management.

General Anxiety/Worry. Endorsement of general anxiety/chronic worry as a key problem focus of their psychotherapy was not significantly associated with either the endorsement $\left(\chi^{2}(1)=.02, p=.89\right)$ or the degree of recalled focus $(F(1,466)=.06, p=.80)$ on positive emotions. Similarly, endorsement of general anxiety/chronic worry was not significantly associated with either the endorsement $(\chi 2(1)=.01, p=.91)$ or the degree of focus $(F(1,417)$ $=.75, p=.39)$ on motivation. Endorsement of general anxiety/chronic worry was not significantly associated with working alliance $\left(\chi^{2}(1)=3.34, p=.07\right)$, yet for those who did report that this was present (45.5\%), endorsing general anxiety was associated with lower 
recalled emphasis ratings, $F(1,281)=7.99, p<.01$. Endorsement of general anxiety was significantly associated with both the endorsement $\left.\left(75.4 \%, \chi^{2}(1)=4.80\right), p<.05\right)$ and greater degree of focus $(F(1,391)=7.78, p<.01)$ on thinking patterns, as well as the endorsement $\left(69.5 \%, \chi^{2}(1)=17.7, p<.05\right)$ and greater degree of focus $(F(1,326)=7.10, p<.01)$ on relaxation training. Also, individuals who endorsed general anxiety were significantly more likely to report attention to stress management $\left(\chi^{2}(1)=16.1, p<.01\right)$, and for those who reported that this was present $(81.2 \%)$, endorsing general anxiety was associated with stronger recalled emphasis ratings, $F(1,397)=36.70, p<.01$.

Panic. Individuals who identified panic as a key problem focus of their psychotherapy were not significantly more likely to report attention to thinking patterns $\left(\chi^{2}(1)=.65, p=0.42\right)$, yet for those who did report that this was present (75.0\%), endorsing panic was associated with stronger recalled emphasis ratings, $F(1,391)=12.8, p<.01$. In addition, endorsement of panic was associated with greater likelihood of recalling relaxation training $\left(76.4 \%, \chi^{2}(1)=7.72, p\right.$ $<.05)$.

Obsessions and Compulsions. Individuals who endorsed obsessions and compulsions as a key problem focus of their psychotherapy were not significantly more likely to report attention to thinking pattern $\left(\chi^{2}(1)=1.23, p=0.27\right)$, but for those who did report that this was present (78.4\%), endorsing obsessions and compulsions was associated with stronger recalled emphasis ratings, $F(1,391)=6.34, p<.01$. Endorsement of obsessions and compulsions was also associated with greater likelihood of recalling relaxation training $\left(77.8 \%, \chi^{2}(1)=5.69, p<.05\right)$. 
Social Anxiety. Individuals who endorsed social anxiety as a key problem focus of their psychotherapy were not significantly more likely to endorse attention to positive emotions $\left(\chi^{2}(1)\right.$ $=.01, p=.91)$ or exhibit differences in the degree of focus on positive emotions, $F(1,466)=1.38$, $p=.24)$. Although endorsement of social anxiety was not significantly associated with recalled focus on confronting difficult situations $\left(\chi^{2}(1)=2.16, p=.14\right)$, for those who did report that this was present $(81.7 \%)$, endorsing social anxiety was associated with stronger recalled emphasis ratings, $F(1,433)=6.87, p<.01$. Endorsement of social anxiety was not significantly associated with thinking patterns $\left(\chi^{2}(1)=3.30, p=.07\right)$, yet for those who did report that this was present (77.1\%), endorsing social anxiety was associated with stronger recalled emphasis ratings, $F(1$, $391)=27.9, p<.01$. Additionally, endorsement of social anxiety was significantly associated with both the endorsement $\left(54.2 \%, \chi^{2}(1)=5.34, p<.05\right)$ and the degree of recalled focus $(F(1,248)=11.3, p<.01)$ on social skills training. In addition, for those who did recall attention to stress management (69.4\%), social anxiety was associated with stronger recalled emphasis, $F(1,397)=7.80, p<.01$.

Relationship and Family. Endorsement of relationship/family problems was not significantly associated with attention to motivation $\left(\chi^{2}(1)=3.28, p=.07\right)$, but for those who did report that this was present $(71.1 \%)$, endorsing relationship problems was associated with less recalled emphasis, $F(1,417)=4.2, p<.01$. Similarly, endorsement of relationship problems was not significantly associated with thinking patterns $\left(\chi^{2}(1)=.43, p=.51\right)$, yet for those who did report that this was present $(71.5 \%)$, endorsing relationship problems was associated with 
less recalled emphasis, $F(1,391)=4.24, p<.01$.

Eating Problems. Individuals who endorsed eating problems as a key focus of their psychotherapy were not significantly more likely to report attention to positive emotions $\left(\chi^{2}(1)\right.$ $=.00, p=0.99)$, yet for those who did report that this was present $(82.2 \%)$, endorsing eating problems was associated with stronger recalled emphasis, $F(1,466)=15.5, p<.01$. Endorsement of eating problems was not significantly associated with encouragement to confronting avoided situations $\left(\chi^{2}(1)=1.48, p=.22\right)$, but for those who did report that this was present $(84.1 \%)$, endorsing eating problems was associated with stronger recalled emphasis, $F(1$, $433)=6.59, p<.01$. Endorsement of eating problems was not significantly associated with thinking patterns $\left(\chi^{2}(1)=3.32, p=.07\right)$, but for those who did report that this was present $(82.2 \%)$, endorsing eating problems was associated with stronger recalled emphasis, $F(1,391)=$ 18.6, $p<.01$. In addition, endorsement of eating problems was not significantly associated with stress management $\left(\chi^{2}(1)=2.02, p=.16\right)$, but for those who did report that this was present $(81.4 \%)$, endorsing eating problems was associated with stronger recalled emphasis ratings, $F(1$, $397)=4.85, p<.01$.

Substance Use. Endorsement of substance problems was associated with lower likelihood of recalling a focus on positive emotions $\left(60.0 \%, \chi^{2}(1)=8.77, p<.05\right)$; similarly, this was associated with lower likelihood of recalling a focus on stress management $\left(52.0 \%, \chi^{2}(1)=\right.$ $5.21, p<.05)$. Endorsement of substance problems was significantly negatively associated with both the likelihood of endorsement $\left.\left(32.0 \%, \chi^{2}(1)=7.79\right), p<.05\right)$ and the degree of reported 
focus $(F(1,326)=6.14, p<.01)$ on relaxation training.

\section{Associations between Recalled Elements and Current Functioning}

Finally, we conducted a series of one-way ANOVAs with EBP element as the independent variable and the current symptom and functioning scales as the dependent variable. Primary results are reported in Table 4. Individuals who endorsed attention to changing thinking patterns as being present during current or previous psychotherapy had higher current DOCS scores $(F(1,664)=7.31, p<.01)$, indicating higher current severity. Individuals who endorsed focusing on the therapeutic relationship evidenced lower current IIP total scores $(F(1,565)=5.65$, $p<.05)$ and higher current SVS scores $(F(1,564)=7.25, p<.01)$, indicating less current interpersonal distress and greater subjective vitality. In addition, attention to the working alliance was associated with lower current PHQ-9 scores $(F(1,565)=4.12, p<.05)$, which indicates lower depression severity.

Individuals who endorsed relaxation training in their current or previous therapy had higher current DOCS $(F(1,555)=11.71, p<.01)$ and PDSS scores $(F(1,556)=7.09, \mathrm{p}<.01)$, indicating higher severity in both domains. However, this was also associated with higher current SVS scores $(F(1,555)=6.08, p<05)$, implying greater subjective vitality. Individuals who endorsed social skills training as a focus of their psychotherapy had lower Negative Affect scores $(F(1,561)=4.51, p<.05)$, yet they had higher panic severity scores on the PDSS $(F(1,561)=$ $5.62, p<.05)$. Individuals who endorsed the presence of stress management in their current or previous therapy had higher current GAD-7 $(F(1,563)=4.05, \mathrm{p}<.05)$ and PHQ-9scores 
$(F(1,562)=5.73, \mathrm{p}<.05)$, indicating higher current worry and depression severity. However, endorsement of stress management was associated with higher flourishing scores on the FS $(F(1,562)=4.16, p<.05)$, indicating better functioning on this subjective life quality domain. Finally, we failed to observe significant associations between endorsement of psychotherapies that included attention to positive emotions, motivation, or confrontation of avoided feelings and situations and current symptoms and function (all $p \mathrm{~s}>.05$ ).

\section{Discussion}

In order to examine what is and is not happening in routine psychotherapy from the client's perspective, this study examined current and former psychotherapy participants' recollections of what took place during their therapy as it pertains to select EBP elements. In addition, we explored participant factors that might be associated with the presence/absence of EBP elements, as well as the degree of focus on such elements. Information on the presence or absence of EBP elements in routine psychotherapy provides genera evidence of the quality of services being offered in diverse community settings.

Overall, routine psychotherapy clients recalled receiving a variety of EBP elements, the most common being a focus on positive emotions. Perhaps with the exception of CBT manuals for major depressive disorder (e.g., Segal, Williams, \& Teasdale, 2002; Seligman et. al., 2006) and the Unified Protocol for the Transdiagnostic Treatment of Emotional Disorders (UP; Barlow et al., 2011), which explicitly address positive emotions, existing treatment protocols tend to emphasize the reduction of negative emotions and related symptoms. Some research supports the 
existence of two main cognitive-affective systems, including the approach system that connects to positive emotions and the withdrawal system that helps people avoid aversive situations by generating negative/inhibitory emotions (Dunn, 2012). The pathology of depression includes disturbed negativity and diminished interest or pleasure (Castonguay \& Oltmanns, 2013). Current clinical practice appears to emphasize disturbances of negative affect, but deficits in positive affect have received less attention. Interestingly, participants who identified depression as a key problem were not more likely to recall a focus on positive emotions, yet if such a focus was endorsed, positive emotions received relatively frequent attention. These results are encouraging because they imply that other sources of knowledge (i.e., beyond what is or is not codified in specific manuals) pertaining to the role of positive emotions (e.g., Brown \& Barlow, 2009) may be impacting what is delivered in routine practice. Studies indicate that a focus on enhancing positivity can give rise to better treatment outcomes (Dunn, 2012; Gallagher et al., 2013; Seligman, Schulman, \& Tryon, 2007), and clients who have better regulation of positive emotions tend to experience improved functioning (Carl et. al., 2013).

The next most frequently endorsed EBP element was confrontation of avoided situations, which can be conceptualized as a shared specific intervention within the exposure domain. This is not surprising given the prevalence of identified anxiety and related disorders in this sample; most evidence-based treatments for these problem domains include some exposure component (Nathan \& Gorman, 2015). However, only endorsement of social anxiety, specifically, was associated with increased attention to confrontation of avoided situations, thoughts, and feelings. 
Given the identified importance of exposure in evidence based treatments for social anxiety (e.g., Hope, Heimberg, \& Turk, 2010), this is uniquely encouraging; interestingly, exposure-based elements may be so pervasive that problem domain is an ineffective predictor of its presence or absence in routine treatment. Interestingly, female participants were more likely to recall the implementation of such exposure-based strategies. Although speculative, child and youth females are more likely to be diagnosed with "internalizing" disorders (anxiety, depressive, and somatic symptom disorders characterized by behavioral inhibition; Eaton et al., 2012), and exposure-based interventions are more likely to be used for these types of problems. Although not a primary focus of this study, we conducted an exploratory chi-square analysis, which revealed that identified females were more likely than males to endorse suffering from depressive symptoms $(p<.01)$, general anxiety/worry $(p<.01)$, panic $(p<.01)$, and eating problems $(p<.01)$.

The importance of developing and maintaining a positive working alliance, as well as attention to disruptions in the alliance, is a common EBP element (Castonguay et al., 2010; England et al., 2015). Fewer than 50\% of the sample endorsed specific attention to the working alliance in their current or past psychotherapies. However, endorsement of the presence of this element was associated better current interpersonal functioning, higher levels of subjective vitality, and lower depression scores. It is critical to acknowledge that the study design does not allow for causal interpretations, relied on participants' retrospective recall of their therapies, and involved technically a non-clinical sample. Nevertheless, the number and consistency in 
direction of the results for this element are intriguing. In a practice-research network (PRN) study of routine psychotherapy clients' perceived helpful and hindering psychotherapy session events, events involving the working alliance were relatively infrequent. However, session events involving the working alliance evidenced many of the highest degree of helpfulness scores compared to other types of events (e.g., gaining self-awareness). This result implied that although attention to the therapeutic relationship is a low base rate event, when it does occur, it can have a powerful impact on the client's experience of psychotherapy. The results of the present study appear to be consistent with this interpretation.

However, the presence or frequency of an EBP element was not consistently associated with better current functioning. Just as we cannot draw clear causal conclusions about the presence of an element and current positive functioning, we cannot conclude with certainty that lower levels of present functioning are directly, or indirectly, associated with the presence of particular EBP elements. Such individuals with more impaired functioning may have a history of greater impairment or chronicity. For example, the presence of relaxation training and stress management strategies were associated with increased severity in some domains, such as panic, obsessions and compulsions, and worry. Although speculative, these strategies are most necessary to implement with clients who are experiencing more general distress and basic emotion regulation deficits. When a client is not ready or able to engage in prolonged exposure, for example, the therapist may spend more time on basic stress reduction and coping strategies (Craske \& Barlow, 2007). Such relationships may be an indication of a subset of participants 
with histories of more pronounced or chronic maladaptive self-regulation.

In summation, overall, routine psychotherapy clients recalled receiving a variety of EBP elements. The presence or absence of a recalled EBP element was, in some cases, associated with endorsement of a specific problem domain, and the recall of receiving an EBP element was not consistently related to better current functioning. Several study limitations must be noted when interpreting the above results.

\section{Limitations}

Although current and/or previous experience in psychotherapy or counseling was the primary eligibility criterion, the sample cannot be characterized as a clinical sample as these individuals were not recruited at the time of pursuing treatment or directly following a specific, identified course of treatment. In line with this, on average, participants did not score in the clinical range on established measures of symptoms and functioning. Observed differences in severity must be understood as relative differences within a non-clinical-to-mild clinical range. Furthermore, the study involved a convenience sample of university students; consequently, the results may not generalize to older treatment receiving individuals.

Although this study may reinforce the view that researchers need to place a stronger emphasis on the client's perspective, it focused on participants' retrospective recall of treatment events, rather than gold standard independent observational assessments. Granted, such methods may not be feasible to implement on a large scale; however, responses' were likely subject to memory biases. In some ways, the chosen method mimicked the often cited Consumer Reports 
Study on the effectiveness of psychotherapy spearheaded by Seligman (1995), which involved clients' retrospective recall of treatment process and helpfulness. This represents a clear methodological limitation, yet one can also argue that the client's perception and experience of the psychotherapy, even when biased, is valuable information.

In addition, EBP elements were intentionally written in jargon-neutral way to avoid potential confusion, and the emphasis on shared specific elements involves an intermediate level of abstraction when referring to clinical strategies (Goldfried, 1980). However, in an effort to streamline a relatively lengthy assessment, we did not provide long-form descriptions or examples of each EBP element. Consequently, participants may have varied in their interpretation of the items. The impact of such variability may have been mitigated by the relatively large sample size.

Finally, a large number of significance tests were conducted and reported, resulting in likely inflation of Type I error. We elected not to apply corrections to inferential tests due to the highly exploratory nature of this study. As such, significant results should be interpreted cautiously, particularly those identified as significant above the $p=01$ level.

\section{Conclusions}

The field has become increasingly interested in client-centered care and prizing the client's perspective as it relates to treatment process and decision making (Castonguay et al., 2010; Fisher \& Boswell, 2016). We are unaware of research that has directly surveyed psychotherapy and counseling client's perceptions of the presence/absence, and the degree to 
which, shared specific and common EBP elements (England et al., 2015) were a focus of their treatment. Generally speaking, the results are somewhat encouraging regarding the quality of care in routine treatment. Future research should continue to focus on clients' experience of psychotherapy elements, including what was/is most and least helpful, to assist in the refinement and implementation of EBPs, and thereby further clinical innovation. In addition, rather than examine elements independently, future research should examine the pattern or constellation of elements within given treatment episodes. This could potentially inform how EBP elements might be integrated together into a comprehensive treatment approach for commonly occurring problem domains and comorbidities. The potential to improve treatment outcomes from psychological disorders through addressing EBP elements has significant clinical implications.

\section{References}


Abramowitz, J. S., Deacon, B. J., Olatunji, B. O., Wheaton, M. G., Berman, N. C., Losardo, D., Timpano, K. R., McGrath, P. B., Riemann, B. C., Adams, T., Björgvinsson, T., Storch, E. A., \& Hale, L. R. (2010). Assessment of obsessive-compulsive symptom dimensions: development and evaluation of the dimensional obsessive-compulsive scale. Psychological Assessment, 22, 180-198.

Barlow, D. H., Farchione, T. J., Fairholme, C. P., Ellard, K. K., Boisseau, C. L., \& Allen, L. B. (2011). Unified Protocol for Transdiagnostic Treatment of Emotional Disorders: Therapist guide. New York: Oxford University Press.

Barlow, D.H., Sauer, S., Carl. J., Ellard, K.K., \& Bullis, J.R. (2014). The nature, diagnosis, and treatment of neuroticism: Back to the future. Clinical Psychological Science, 2, 344-365. doi: $10.1177 / 2167702613505532$

Boswell, J.F. (2013). Intervention strategies and clinical process in transdiagnostic cognitive-behavioral therapy. Psychotherapy, 50, 381-386. doi: 10.1037/a0032157

Boswell, J.F., Gallagher, M.W., Sauer-Zavala, S.E., Bullis, J., Gorman, J.M., Shear, M.K., Woods, S.W., \& Barlow, D.H. (2013). Patient characteristics and variability in adherence and competence in cognitive-behavioral therapy for panic disorder. Journal of Consulting and Clinical Psychology, 81, 443-454. doi: 10.1037/a0031437

Brown, T.A. (2007). Temporal course and structural relationships among dimensions of temperament and DSM-IV anxiety and mood disorders. Journal of Abnormal Psychology, 116, 313-328. 
Brown, T.A. \& Barlow, D.H. (2009). A proposal for a dimensional classification system based on the shared features of the DSM-IV anxiety and mood disorders: Implications for assessment and treatment. Psychological Assessment, 21, 256-271.

Brown, T. A., Campbell, L. A., Lehman, C. L., Grisham, J. R., \& Mancill, R. B. (2001). Current and lifetime comorbidity of the DSM-IV anxiety and mood disorders in a large clinical sample. Journal of Abnormal Psychology, 110, 49-58.

Brownson, R.C., Colditz, G.A., Proctor, E.K. (Eds.) (2012). Dissemination and implementation research in health: Translating science to practice. New York: Oxford University Press.

Carl, J.R., Fairholme, C.P., Gallagher, M.W., Thompson-Hollands, J., \& Barlow, D.H. (2014). The effects of anxiety and depressive symptoms on daily positive emotion regulation. Journal of Psychopathology and Behavioral Assessment, 36, 224-236. doi $10.1007 / \mathrm{s} 10862-013-9387-9$

Castonguay, L.G., \& Beutler, L.E. (Eds.) (2006). Principles of therapeutic change that work. New York, NY: Oxford University Press.

Castonguay, L. G., Boswell, J. F., Zack, S., Baker, S., Boutselis, M., Chiswick, N., Damer, D., Hemmelstein, N., Jackson, J., Morford, M., Ragusea, S., Roper, G., Spayd, C., Weiszer, T., Borkovec, T. D., \& Grosse Holtforth., M. (2010). Helpful and hindering events in psychotherapy: A practice research network study. Psychotherapy: Theory, Research, Practice, and Training, 47, 327-344.

Castonguay, L.G., Constantino, M.J., Boswell, J.F., \& Kraus, D. (2010). The therapeutic alliance: 
Research and theory. In L. Horowitz, \& S. Strack (Eds.), Handbook of interpersonal psychology: Theory, research, assessment, and therapeutic interventions (pp. 509-518). New York, NY: John Wiley \& Sons.

Castonguay, L.G., \& Oltmanns, T.F. (Eds.) (2013). Psychopathology: From science to clinical practice. New York: Guilford Press.

Craske, M. G. \& Barlow, D. H. (2007). Mastery of your anxiety and panic: Therapist guide. University Press.

Dempster, A.P., Laird, N.M., \& Rubin, D.B. (1977). Maximum likelihood from incomplete data via the EM algorithm. Journal of the Royal Statistical Society: Series B, 39, 1-38.

Diener, E., Wirtz, D., Biswas-Diener, R., Tov, W., Kim-Prieto, C, Choi, D., \& Oishi, S. (2009). New measures of well-being. In E. Diener (Ed.), Assessing well-being: The collected works of Ed Diener (pp. 247 - 266). The Netherlands: Springer. doi:

$10.1007 / 978-90-481-2354-412$

Dunn, B. D. (2012). Helping depressed clients reconnect to positive emotion experience: current insights and future directions. Clinical psychology and psychotherapy, 19, 326-340.

Eaton, N.R., Keyes, K.M., Krueger, R.F., Balsis, S., Skodol, A.E., Markon, K.E., Grant, B.F., \& Hasin, D.S. (2012). An invariant dimensional lability model of gender differences in mental disorder prevalence: Evidence from a national sample. Journal of Abnormal Psychology, 12, 282-288. doi: 10.1037/a0024780

England, M.J., Butler, A.S., \& Gonzalez, M.L. (Eds.) (2015). Psychosocial interventions for 
mental and substance abuse disorders: A framework for establishing evidence-based standards. Washington, D.C.: National Academies Press.

Fisher, A.J., \& Boswell, J.F. (2016). Enhancing the personalization of psychotherapy with dynamic assessment and modeling. Assessment, 23, 496-506. doi:10.1177/1073191116638735

Foa, E. B., Chrestman, K., \& Gilboa-Schechtman, E. (2008). Prolonged exposure therapy for adolescents with PTSD. New York, NY: Oxford University Press

Friedman, K., Ramirez, A. L., Murray, D., Anderson, L. K., Cusack, A., Boutelle, K. N., \& Kaye, W. (2016). A narrative review of outcome studies for residential and partial hospital-based treatment of eating disorders. European Eating Disorders Review, 24(4), 263-276. doi:10.1002/erv.2449

Gallagher, M. W., Sauer-Zavala, S. E., Boswell, J. F., Carl, J. R., Bullis, J., Farchione, T. J., \& Barlow, D. H. (2013). The impact of the unified protocol for emotional disorders on quality of life. International Journal of Cognitive Therapy, 6, 2013.

Garland, A.F., \& Schoenwald, S.K. (2013). Use of effective and efficient quality control methods to implement psychosocial interventions. Clinical Psychology Science and Practice, 20(1), 33-43.

Goldfried, M.R. (1980). Toward a delineation of therapeutic change principles. American Psychologist, 35, 991-999. 
Goisman, R.M., Warshaw, M.G., \& Keller, M.B. (1999). Psychosocial treatment prescriptions for generalized anxiety disorder, panic disorder, and social phobia, 1991-1996. American Journal of Psychiatry, 156, 1819-1821. doi: 10.1176/ajp.156.11.1819

Heimberg, R. G., Horner, K. J., Juster, H. R., Safren, S. A., Brown, E. J., \& Schneier, F. R. (1999). Psychometric properties of the Liebowitz social anxiety scale. Psychological Medicine, 29(1), 199-212.

Hope, D. A., Heimberg, R. G., \& Turk, C. L. (2010). Managing Social Anxiety: A Cognitive-Behavioral Therapy Approach: Workbook. New York: Oxford University Press.

Horowitz, L. M., Alden, L. E., Wiggins, J. S., \& Pincus, A. L. (2000). Inventory of Interpersonal Problems. London: Psychological Corporation.

Horvath, A. O., Del Re, A., Flückiger, C., \& Symonds, D. (2011). Alliance in individual psychotherapy. In J. C. Norcross (Ed.), Psychotherapy relationships that work: Evidence-based responsiveness (2nd ed., pp. 25-69). New York, NY: Oxford University Press.

Kazdin, A.E. (2008). Evidence-based treatment and practice: new opportunities to bridge clinical research and practice, enhance the knowledge base, and improve patient care. American Psychologist, 63, 146-159.

Kroenke K, Spitzer RL, Williams JBW. (2001). The PHQ-9 — validity of a brief depression severity measure. Journal of General Internal Medicine. 16, 606-13. 
Lambert, M.J. (Ed.). Bergin \& Garfield's handbook of psychotherapy and behavior change (6th ed.). New York, NY: John Wiley \& Sons.

Liebowitz, M. R. (1987). Social phobia. Modern Problems of Pharmacopsychiatry, 22, 141-173.

Nathan, P. (Ed.), \& Gorman, J. (Ed.). (2015). A guide to treatments that work (4 ${ }^{\text {th }}$ ed.). New York: Oxford University Press.

Ryan, R.M., \& Frederick, C. (1997). On energy, personality and health: Subjective vitality as a dynamic reflection of well-being. Journal of Personality, 65, 529-565.

Seligman, M.E.P. (1995). The effectiveness of psychotherapy: The Consumer Reports Study. American Psychologist, 12, 965-974.

Seligman, M.E.P., Rashid, T., \& Parks, A. C. (2006). Positive psychotherapy. American Psychologist, 61, 774-788.

Seligman, M.E.P., Schulman, P., \& Tryon, A. (2007). Group prevention of depression and anxiety symptoms. Behavior Research and Therapy, 45, 1111-1126.

Segal, Z.V., Williams, J.M.G., \& Teasdale, J.D. (2002). Mindfulness-based cognitive therapy for depression: a new approach to preventing relapse. New York: Guilford Press.

Shear MK, Rucci P, Williams J, Frank E, Grochocinski V, Vander Bilt J, Wang T. (2001). Reliability and validity of the Panic Disorder Severity Scale: Replication and extension. Journal of Psychiatric Research, 35, 293-296.

Spitzer, R.L., Kroenke, K., Williams, J.B., Lowe, B. (2006). A brief measure for assessing generalized anxiety disorder: the GAD-7. Archives of Internal Medicine, 166, 1092- 
1097.

Stirman, S.W., Gutner, C.A., Crits-Christoph, P., \& Beidas, R.S. (2015). Relationships between clinician-level attributes and fidelity-consistent and fidelity-inconsistent modifications to and evidence-based psychotherapy. Implementation Science, 10:115.

doi:10.1186/s13012-015-0308-z

Tabachnick, B.G., \& Fidell, L.S. (2013). Using multivariate statistics (6 $6^{\text {th }}$ ed.). Upper Saddle River, NJ: Pearson.

Waller, G., Stringer, H., \& Meyer, C. (2012). What cognitive behavioral techniques do therapists report using when delivering cognitive behavioral therapy for the eating disorders. Journal of Consulting and Clinical psychology, 80, 171-175.

Watson, D., Clark, L. A., \& Carey, G. (1988a). Positive and negative affectivity and their relation to anxiety and depressive disorders. Journal of Abnormal Psychology, 97, 346-353.

Watson, D., Clark, L. A., \& Tellegen, A. (1988b). Development and validation of brief measures of positive and negative affect: The PANAS Scales. Journal of Personality and Social Psychology, 47, 1063-1070. 
Table 1. Means, standard deviations, possible ranges, and internal consistencies

\begin{tabular}{lllll}
\hline Measure & Total Score M & SD & Possible Range & Cronbach's $\alpha$ \\
\hline LSAS & 51.6 & 27.2 & $0-144$ & .96 \\
PANAS_PA & 28.1 & 8.4 & $10-50$ & .90 \\
PANAS_NA & 24.1 & 7.9 & $10-50$ & .86 \\
IIP-32 & 39.2 & 23.9 & $0-128$ & .93 \\
DOCS & 16.7 & 12.1 & $0-80$ & .93 \\
GAD-7 & 7.8 & 5.6 & $0-21$ & .91 \\
PDSS-SR & 7.8 & 3.5 & $0-20$ & .87 \\
PHQ-9 & 8.8 & 6.3 & $0-27$ & .89 \\
SVS & 26.3 & 7.7 & $7-49$ & .76 \\
FS & 40.0 & 10.6 & $8-56$ & .94 \\
\hline
\end{tabular}

Note. LSAS $=$ Liebowitz Social Anxiety Scale; PANAS_PA $=$ Positive and Negative Affect Schedule_Positive Affect; PANAS_NA $=$ Positive and Negative Affect Schedule_Negative Affect; IIP-32 = Inventory of Interpersonal Problems; DOCS $=$ Dimensional Obsessive-Compulsive Scale; GAD-7 = General Anxiety Disorder-7 Scale; PDSS-SR $=$ Panic Disorder Severity Scale; PHQ-9 = Patient Health Questionnaire; SVS = Subjective Vitality Scale; FS $=$ Flourishing Scale 
Table 2. Evidence-based practice (EBP) endorsement frequencies and degree of focus descriptives

\begin{tabular}{|c|c|c|}
\hline EBP Element & $\begin{array}{l}\text { Frequency } \\
\text { YES\% (n) }\end{array}$ & $\begin{array}{c}\text { If YES } \\
\text { Degree } M(\mathrm{SD}) \\
\text { Range }=1-4\end{array}$ \\
\hline Positive Emotions & $79.8(470)$ & $2.27(.71)$ \\
\hline Avoidance & $74.0(436)$ & $2.45(.83)$ \\
\hline Motivation & $71.6(422)$ & $2.30(.75)$ \\
\hline Stress Management & $69.1(407)$ & $2.54(.87)$ \\
\hline Thoughts & $67.6(398)$ & $2.52(.84)$ \\
\hline Relaxation & $55.9(329)$ & $2.39(.89)$ \\
\hline Relationship & $48.4(285)$ & $2.03(.76)$ \\
\hline Social Skills & $43.0(253)$ & $2.28(.87)$ \\
\hline
\end{tabular}


Table 3. Evidence-Based Practice Elements by Demographics

\begin{tabular}{lcccc|cccc}
\hline $\begin{array}{l}\text { EBP } \\
\text { Element }\end{array}$ & $\begin{array}{c}\text { \% YES } \\
\text { Female }\end{array}$ & $\begin{array}{c}\text { M YES } \\
\text { Male }\end{array}$ & $\chi^{2}$ & $F$ & $\begin{array}{c}\text { \% YES } \\
\text { Majority }\end{array}$ & $\begin{array}{c}\text { \% YES } \\
\text { Majority }\end{array}$ & $\chi^{2}$ & $F$ \\
\hline $\begin{array}{l}\text { Positive } \\
\text { Emotions } \\
\text { Avoidance }\end{array}$ & 81.5 & 83.1 & 0.25 & 0.13 & 81.3 & 83.3 & 0.39 & 3.86 \\
Motivation & 79.9 & 71.9 & $4.96^{*}$ & $4.43^{*}$ & 75.8 & 77.6 & 0.27 & 3.55 \\
$\begin{array}{l}\text { Stress } \\
\text { Management }\end{array}$ & 75.7 & 66.8 & $5.34^{*}$ & 1.22 & 71.2 & 73.1 & 0.27 & 0.09 \\
Thoughts & 72.7 & 67.0 & 2.15 & 2.51 & 69.6 & 71.1 & 0.15 & 1.42 \\
Relaxation & 58.1 & 60.0 & 0.21 & 0.01 & 56.5 & 61.8 & 1.58 & 0.45 \\
Relationship & 47.9 & 53.2 & 1.54 & $6.42^{*}$ & 46.7 & 54.3 & 3.30 & $4.57^{*}$ \\
Social Skills & 40.2 & 51.5 & $7.09^{*}$ & 0.13 & 39.7 & 51.2 & $7.47^{*}$ & 2.28 \\
\hline
\end{tabular}

Note: $* \mathrm{p}<.05 ; \mathrm{n}$ 's varied for each element $(\mathrm{n}=562-575) . F$-tests conducted to compare extent of focus of element during psychotherapy ( $1=$ Rarely to $4=$ Extensively) for those who endorsed specific practice element. 
Table 4. Presence/Absence of EBP Element and Current Functioning ANOVA Results

\begin{tabular}{|c|c|c|c|c|c|c|c|c|}
\hline Measure/ & & & & EPB & & & & \\
\hline DV & & & & Element & & & & \\
\hline & Positive & Motivation & Relationship & Avoidance & Thoughts & Relaxation & Social & Stress \\
\hline & Emotion & $F$ & $F$ & $F$ & $F$ & $F$ & Skills & Management \\
\hline & $F$ & (df) & (df) & (df) & (df) & (df) & $F$ & $F$ \\
\hline & (df) & & & & & & (df) & (df) \\
\hline LSAS & 0.00 & 0.61 & 1.01 & 3.19 & 3.51 & 0.11 & 0.88 & 1.28 \\
\hline & $(1,553)$ & $(1,549)$ & $(1,550)$ & $(1,550)$ & $(1,547)$ & $(1,541)$ & $(1,545)$ & $(1,546)$ \\
\hline $\mathrm{PA}$ & 0.20 & 1.15 & 0.31 & 0.25 & 0.86 & 1.67 & 0.51 & 0.34 \\
\hline & $(1,569)$ & $(1,565)$ & $(1,566)$ & $(1,566)$ & $(1,563)$ & $(1,556)$ & $(1,561)$ & $(1,562)$ \\
\hline NA & 0.89 & 2.78 & 2.40 & 1.15 & 0.02 & 0.01 & $4.51^{*}$ & 0.74 \\
\hline & $(1,569)$ & $(1,565)$ & $(1,566)$ & $(1,566)$ & $(1,563)$ & $(1,556)$ & $(1,561)$ & $(1,562)$ \\
\hline IIP & 0.00 & 1.39 & $5.65^{*}$ & .034 & 2.10 & 1.00 & 0.01 & 0.07 \\
\hline & $(1,567)$ & $(1,564)$ & $(1,565)$ & $(1,566)$ & $(1,563)$ & $(1,556)$ & $(1,561)$ & $(1,562)$ \\
\hline DOCS & 0.00 & 0.68 & 1.60 & 0.56 & $7.31 * *$ & $11.71^{* *}$ & 3.73 & 3.10 \\
\hline & $(1,567)$ & $(1,563)$ & $(1,564)$ & $(1,565)$ & $(1,664)$ & $(1,555)$ & $(1,560)$ & $(1,561)$ \\
\hline GAD & 0.03 & 0.00 & 1.79 & 3.33 & 3.20 & 3.94 & 1.05 & $4.05^{*}$ \\
\hline & $(1,569)$ & $(1,565)$ & $(1,566)$ & $(1,567)$ & $(1,564)$ & $(1,557)$ & $(1,562)$ & $(1,563)$ \\
\hline PDSS & 0.10 & 0.86 & 2.55 & 1.17 & 2.55 & $7.09 * *$ & $5.62 *$ & 2.16 \\
\hline & $(1,568)$ & $(1,564)$ & $(1,565)$ & $(1,566)$ & $(1,563)$ & $(1,556)$ & $(1,561)$ & $(1,562)$ \\
\hline PHQ & 0.09 & 0.57 & $4.12^{*}$ & 0.40 & 1.84 & 0.07 & 0.01 & $5.73^{*}$ \\
\hline & $(1,568)$ & $(1,564)$ & $(1,565)$ & $(1,566)$ & $(1,563)$ & $(1,556)$ & $(1,561)$ & $(1,562)$ \\
\hline SVS & 1.58 & 1.70 & $7.25^{* *}$ & 0.95 & 0.62 & $6.08 *$ & 1.53 & .045 \\
\hline & $(1,567)$ & $(1,563)$ & $(1,564)$ & $(1,565)$ & $(1,562)$ & $(1,555)$ & $(1,560)$ & $(1,561)$ \\
\hline
\end{tabular}




\begin{tabular}{ccccccccc}
\hline FS & 1.95 & 2.03 & 3.61 & 1.13 & 0.35 & 2.54 & 0.56 & $4.16^{*}$ \\
& $(1,568)$ & $(1,564)$ & $(1,565)$ & $(1,566)$ & $(1,563)$ & $(1,556)$ & $(1,561)$ & $(1,562)$ \\
& & & & & & & \\
\hline
\end{tabular}

Note. $\quad$ LSAS $=$ Liebowitz Social Anxiety Scale; PA = Positive Affect; NA = Negative Affect; IIP = Inventory of Interpersonal Problems; DOCS = Dimensional Obsessive-Compulsive Scale; GAD $=$ General Anxiety Disorder; PDSS = Panic Disorder Severity Scale; PHQ = Patient Health Questionnaire; SVS = Subjective Vitality Scale; FS = Flourishing Scale. 